\title{
1 Environmental Colloids and Particles: Current Knowledge and Future Developments
}

\author{
JAMIE R. LEAD \\ Division of Environmental Health and Risk Management, School of Geography, Earth and \\ Environmental Sciences, University of Birmingham, Birmingham B15 2TT, UK \\ KEVIN J. WILKINSON \\ Department of Chemistry, University of Montreal, P.O. Box 6128, Succ. Centre-ville, \\ Montreal, Canada H3C $3 \mathrm{J7}$
}

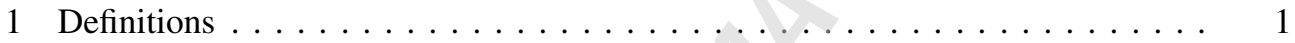

2 The Importance of Size $\ldots \ldots \ldots \ldots \ldots \ldots \ldots \ldots \ldots \ldots \ldots \ldots$

3 Non-size-based measurements of colloids and particles . . . . . . . . . . 9

4 Strategies for advancing our understanding of colloidal systems . . . . . . 10

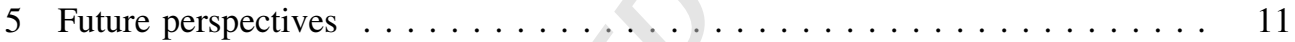

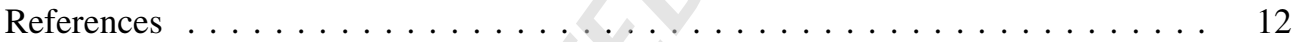

\section{DEFINITIONS}

The precise definition of environmental colloids or environmental colloidal systems was discussed in the first volume of this series [1], but still remains a matter of some debate, years later. In a number of areas, including environmental chemistry, the idea of phase has been excluded from common usage and we now often talk of colloids rather than colloidal systems. This procedure will be followed here, but it should be remembered that environmental colloids are relatively dilute dispersions of solid (sometimes liquid or gas) phases within a water or atmospheric gas phase. Although a great deal of discussion revolves around the exact meanings of the terms 'colloids' and 'particles', to some extent, these problems are trivial, in that they primarily relate to nomenclature differences amongst different disciplines and different researchers that could be avoided by the careful and systematic use of appropriate terms. For instance, a size-based definition was first developed in the field of colloid chemistry [2], however, water engineers frequently use membrane filters with nominal pore sizes of about $5-10 \mu \mathrm{m}$, while aquatic chemists commonly use 0.2 or $0.45 \mu \mathrm{m}$ pore sizes. Even within the broadly defined environmental sciences, different definitions are employed. For example, membranes with a $c a ., 1 \mathrm{~nm}(1 \mathrm{kDa})$ nominal pore

Environmental Colloids and Particles. Edited by K. J. Wilkinson and J. R. Lead (c) 2007 IUPAC 
size are often used to discriminate, somewhat arbitrarily, between the truly dissolved and the non-aqueous phases. Finally, further confusion has recently occurred due to the now common use of terms such as macromolecules and nanoparticles. Generally, macromolecule refers to a small polymeric colloid while the term nanoparticle is also applied to the very smallest colloids [3], usually below $100 \mathrm{~nm}$.

In this chapter and throughout the book, the lower size limit for colloids is set in a similar manner to traditional colloid chemistry, i.e., any organic or inorganic entity large enough to have a supramolecular structure and properties that differ markedly from those of the aqueous phase alone, e.g., possibility of conformational changes or the development of an electrical surface field. This limit coincides with the environmentally relevant condition that aquatic colloids are generally small enough that, in the absence of aggregation, Brownian motion is sufficient to keep them suspended in the water column for long periods (>hours-days). Similarly, the upper size limit corresponds to the point where interfacial phenomena are qualitatively less important due to the smaller relative surface to volume ratio of the colloid/particle, although interfacial phenomena are important in all environmental systems. From the preceding constraints has evolved the more practical IUPAC definition that the colloidal size range will typically have at least one dimension in the $1 \mathrm{~nm}$ to $1 \mu \mathrm{m}$ size range [1,4], while particles are defined as materials whose dimensions are $>1 \mu \mathrm{m}$.

Clearly, there are limitations on the usefulness of these definitions, for both practical and theoretical reasons. Due to both the chemical and physical complexity of environmental colloids and particles and a lack of standardisation of analytical techniques, including fractionation and sizing methods (especially filtration [5]), experimental data can rarely be rigorously related to even the operational IUPAC definitions. For example, it is not yet possible to determine direct, systematic and rigorous relationships between the physicochemical properties of membrane permeates or retentates and their environmental function. The current size-based definitions are essentially operational; greater understanding may be gained from definitions that are based on colloidal structure or function (environmental role), in an analogous manner to biological macromolecules. Unfortunately, it is not yet clear that any such attempt would be successful, again due to the complexity of environmental colloids and particles.

Nevertheless, the search for more fundamental definitions of colloids is in progress [6]. For example, it has been argued that colloids should be defined as those species for which no chemical potential can be defined [7]. In a view that was developed further by Gustafsson and Gschwend [6], Buffle and Leppard considered that colloids were dominated by aggregation processes whereas particles were dominated by sedimentation [8]. According to Gustafsson and Gschwend, a colloid in one water body could behave as a particle in another water body, depending on the precise physicochemistry of the medium. This implies that the distinction between colloids and particles may be both site and time specific. Gustafsson and Gschwend [6] distinguished the colloidal from the dissolved phase by the presence or absence of an internal milieu with properties, such as a dielectric constant, that are substantially different from those of the bulk solution. In such a case, a polyelectrolyte with no internal spaces could be considered as being a dissolved species, although this view has been challenged [9]. Clearly, the requirement for an internal milieu poses some problems and neglects the importance of the surface binding of pollutants. 
Although these definitions are relatively comprehensive, it must be recognised that they are limited when applied to the environment. For example, sedimentation is surely of less importance in groundwaters than it is in surface waters. Second, as noted above, the surface properties of colloids and particles are extremely important. Third, colloids are not at equilibrium but rather exist as a dynamic system with respect to aggregation processes, pollutant binding and biological uptake. Finally, even if the conceptual models provide a good starting point for further understanding the role and impact of colloids and particles, it is currently extremely difficult to validate the models with rigorous analytical results. Despite important progress over the past few decades of research, we are only at the very beginnings of a comprehensive understanding in this field.

\section{THE IMPORTANCE OF SIZE}

As mentioned above, between $1 \mathrm{~nm}$ and $1 \mu \mathrm{m}$, solid-phase materials are dominated by surface properties, including surface area and electrical charge, rather than bulk properties such as the chemical composition of the colloids. Interfacial properties are particularly important at the lower end of the size scale. For example, about $50 \%$ of the mass (or atoms) is found at the surface of a $3 \mathrm{~nm}$ colloid compared with about $5 \%$ of the mass for a $30 \mathrm{~nm}$ colloid [3]. Colloidal surface properties are therefore extremely important to understanding environmental function since colloidal aggregation and the sorption of trace pollutants, nutrients and pathogens are dependent on the nature of the colloid-colloid and colloid-water interfaces [10-12]. At the upper end of the colloidal size scale (ca. $1 \mu \mathrm{m})$, not only do surface properties become less relevant, but also gravitational forces begin to exceed forces due to Brownian motion, with a resulting sedimentation of the particles/aggregates [13].

Historically, filtration through a $0.45 \mu \mathrm{m}$ filter has been used to distinguish the particulate and dissolved phases. The filtration step has also been used to reduce sample complexity, partially to sterilise the dissolved phase through removal of a majority of the microorganisms and to improve analytical sensitivity, e.g. by reducing fouling on electrode or other surfaces. More recently, additional filtration steps have been introduced [5,14] such that filtration is now typically performed with several nominal pore sizes in order to define dissolved, colloidal and particulate phases. Nevertheless, it must be emphasised that these phases are purely operational and are not necessarily related to real differences in structure or to environmental or chemical behaviour. In addition, quantitative comparisons between data sets are difficult due to a variability in the nominal pore sizes that are used and to artefacts that are inherent in the filtration method [4,15-17]. Nonetheless, filtration has produced a wealth of data and greatly advanced our understanding of environmental colloids and particles. However, as stressed later in this volume, confidence in filtration and ultrafiltration data requires that rigorous protocols are implemented, including calculation of mass balances and quantification of the particle size distributions in the sample, retentate and permeate using appropriate microscopic techniques. These verifications are rarely performed and therefore a large proportion of literature data must be critically re-evaluated.

Very little is known, in detail and with accuracy, about the true size distribution of naturally occurring colloids and particles, either as isolated entities or as aggregates, in any environmental compartment. A simplified size distribution of several key biological, organic and mineral phases is given in Figure 1. A key observation from this figure is 


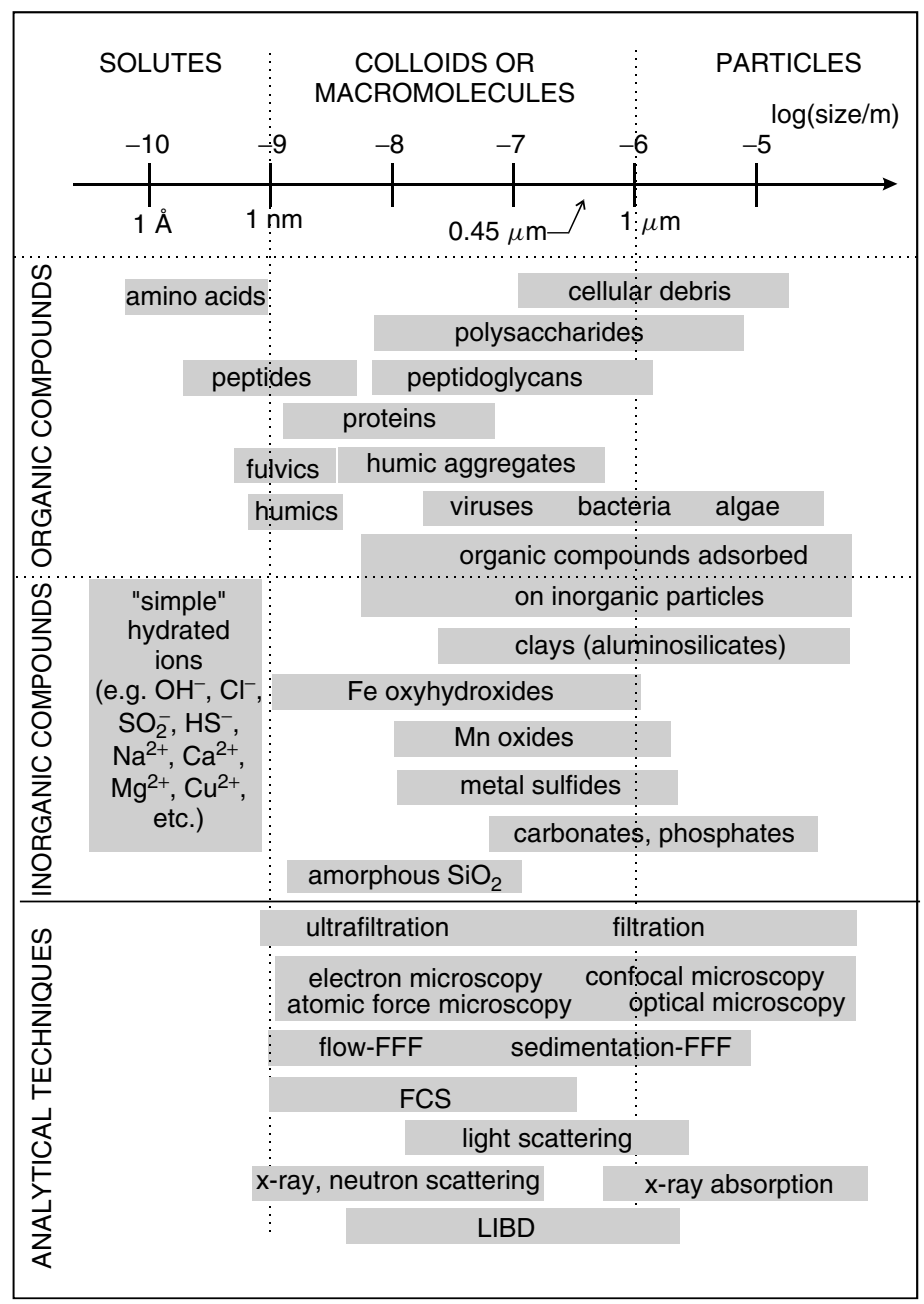

Figure 1. Size distributions of various types of environmental colloids and particles and several of the analytical techniques that are used to characterize them. Abbreviations: $\mathrm{FFF}=$ field-flow fractionation (Chapter 5); FCS = fluorescence correlation spectroscopy (Chapter 11); LIBD = laser-induced breakdown spectroscopy (Chapter 12). Adapted from [1] with permission from Taylor and Francis

that the sizes of each of the apparently homogeneous colloid types are often spread over several orders of magnitude. Furthermore, the categorisation of different colloid types is somewhat artificial since, in natural systems, colloid groups are rarely found in purified forms but most often are components of complex heteroaggregates. Even a 'homogeneous' colloid class such as the humic substances are better described as a complex mixture that includes recognisable biomolecules [18]. Figure 1 also indicates the approximate size ranges in which several of the key colloidal characterisation techniques operate.

Several examples of some colloidal and particulate size distributions, measured using different techniques on unperturbed natural waters, are given in Figure 2. Interestingly, 

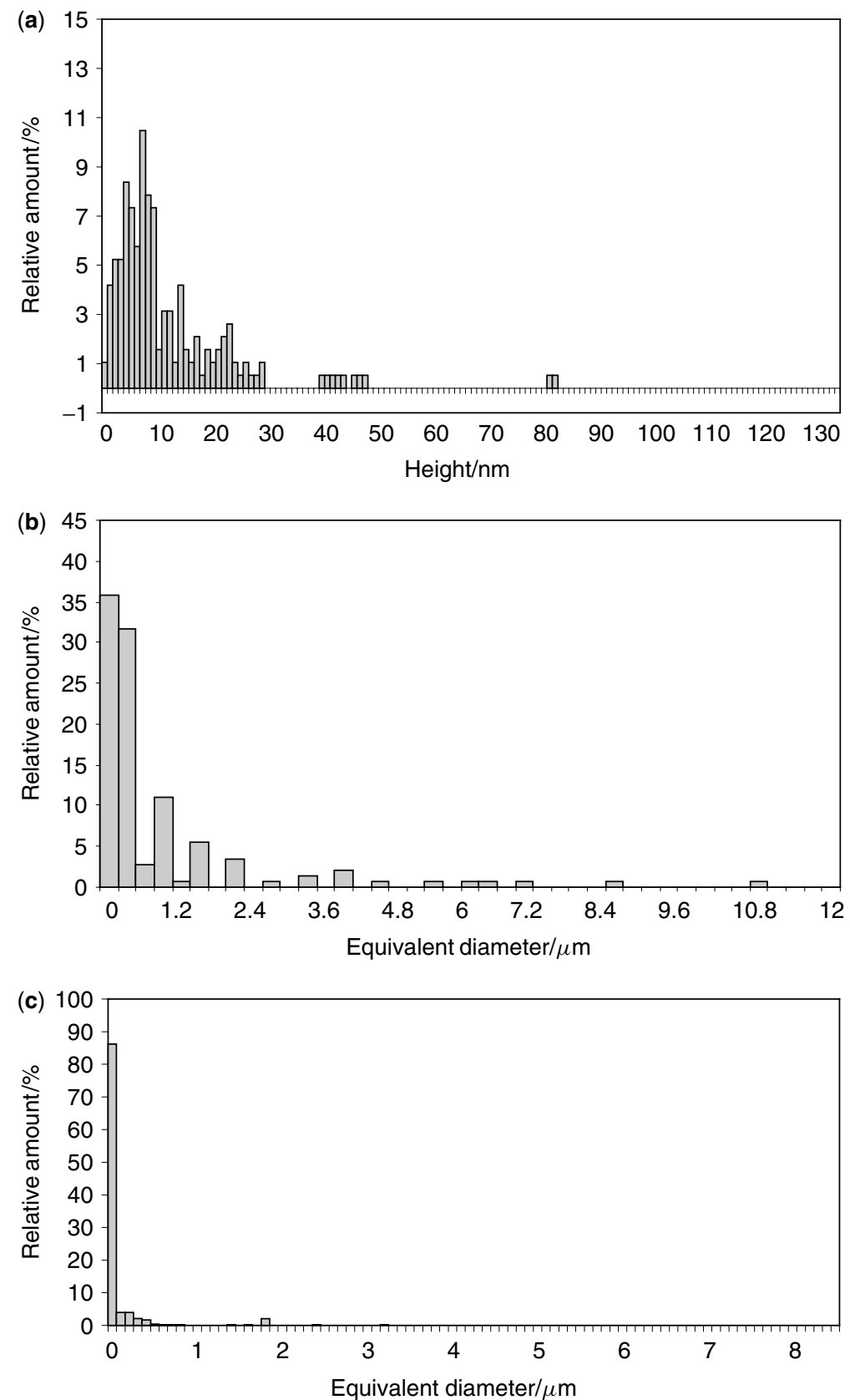

Figure 2. Particle size distributions calculated from AFM, ESEM, SEM [15] and SdFFF [19]. Parts (a)-(c) from Journal of Environmental Monitoring, 2005, 7, 115-121. Reproduced by permission of The Royal Society of Chemistry 
observed sizes are often distributed roughly normally (usually skewed with a long tail) within the analytical window of the technique being employed [15], strongly suggesting an important limitation of the individual techniques and the need to use several characterisation techniques simultaneously, a theme which we will return to later in this chapter. Indeed, Figure 3 shows comparative images using scanning electron microscopy (SEM) and environmental scanning electron microscopy (ESEM) of the same sample with considerably different observed morphologies. The variability of the distributions demonstrates both the difficulty of producing reliable data and the fundamentally different nature of data collected by different techniques. Nevertheless, this situation allows a number of techniques to be applied to produce representative data on the nature of colloids and their interactions.

Although average measures are most often quantified, environmental colloids are always polydisperse, often with undefined size distributions. Size distributions can be determined experimentally by field-flow fractionation (FFF) $[21,22]$, electron microscopy $[15,23,24]$ and atomic force microscopy (AFM) $[15,25,26]$ and calculated from fluorescence correlation spectroscopy (FCS) [27-29] and dynamic light scattering [30]. Nevertheless, the techniques each have different detection limits and detection windows corresponding to different size ranges (Figure 1). Some techniques are simply incapable of measuring accurately the whole range of data on polydisperse samples, a factor which may both skew the data collected and invalidate their interpretation. In addition, size distributions may be related to the mass, number or surface area of the colloids as described by the number-, weight- or z-averages (Table 1). Unfortunately, a large proportion of literature values do not specify which average is determined, in spite of the fact that for polydisperse samples, the different averages will have very different numerical values (Figure 4). Furthermore, most of the colloidal sizing techniques do not measure size directly but rather a different physicochemical property from which the size is derived. For example, whereas physical (number-average) dimensions are determined by microscopic techniques $[15,24]$, diffusion coefficients are generally determined from light scattering, size-exclusion chromatography, flow field-flow fractionation (FIFFF) [21,28] and FCS [27,29]. Charge/size ratios are derived from electrophoretic mobilities [31] and buoyant mass from sedimentation FFF [21] and other centrifugation-based techniques such as analytical ultracentrifugation. Although molecular dimensions and molar masses can be estimated from diffusion coefficients (and eventually electrophoretic mobilities when coupled to titration data), the calculations are based on a large number of (sometimes unwarranted) assumptions (sphericity, permeability, homogeneous charge distribution, absence of aggregates, etc.) [32]. Although each of the above points refers to current and important problems, they will be discussed but not solved in this volume; future good-quality science will need to meet these challenges in appropriate ways.

Consequently, numerous, largely unsupported, assumptions have often been accepted by the environmental sciences community. For instance, the importance of colloids is often ascribed to an increase in specific surface area with decreasing size resulting in the exposure of a greater number of functional groups at the solid-aqueous interface and thus a greater uptake of trace pollutants. Furthermore, sizes are generally held to follow a Pareto or other (e.g. log-normal [33]) distribution (Figure 2). However, the actual high-quality experimental data to support these beliefs (in particular those employing 
(a)
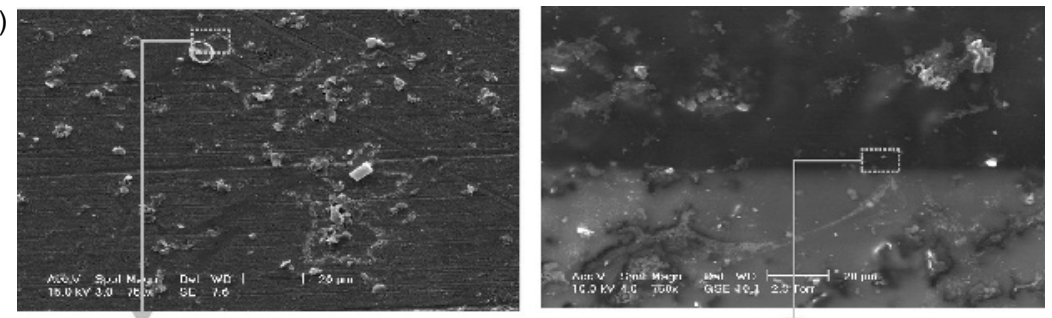

(b)

(c)
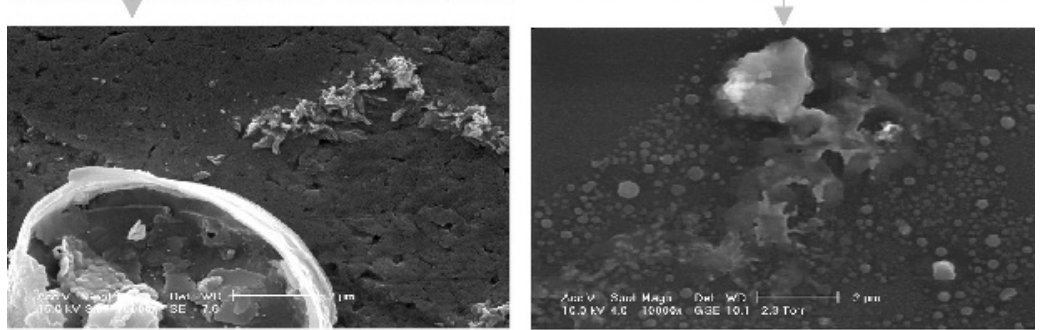

(d)

(e)
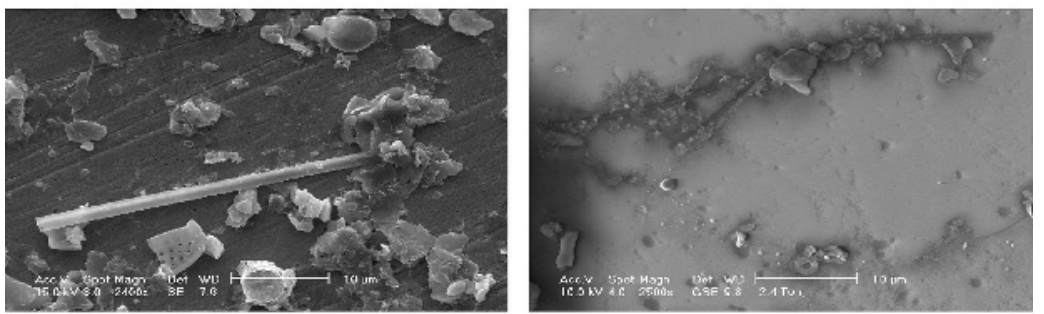

(g)
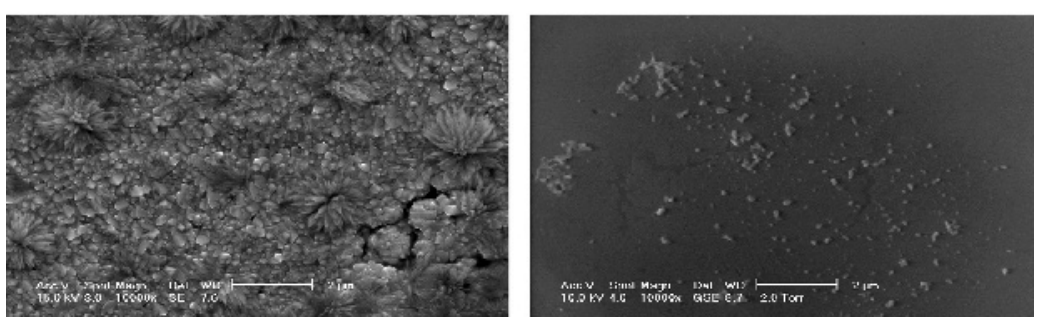

(h)

Figure 3. Images of the Tamar Estuary taken by both SEM (a, c, e, g) and ESEM (b, d, f, h) [20]. The images are taken from water from the same sample bottle, but $\mathrm{a}-\mathrm{b}, \mathrm{c}-\mathrm{d}, \mathrm{e}-\mathrm{f}$ and $\mathrm{g}-\mathrm{h}$ are not image pairs of exactly the same sample. Reprinted from Doucet, F. J., Maguire, L. and Lead, J. R., Size fractionation of aquatic colloids and particles by cross-flow filtration, analysis by scanning electron and atomic force microscopy, Analytica Chimica Acta, 522, 59-71, Copyright 2004, with permission from Elsevier

several different techniques) are extremely small. In addition, it is highly likely that many types of environmental colloids are permeable to both water [34] and trace pollutants so that simply taking into account surface complexation reactions would result in an underestimation of colloidal binding. Indeed, following their adsorption on the surface of the colloidal particle, it is likely, in many cases, that pollutants are taken up into the body of the colloid or particle [35]. A final related point is that very few studies have been performed in situ: most results have been extrapolated from partially 
Table 1. Equations for number-, weight- and $z$-average molar masses and diameters. Several techniques allow for the near direct determination of these average values (e.g. electron or transmission electron microscopy: number-average diameters; fluorescence correlation spectroscopy: number-(single fluorophore) or weight-(several fluorophores) average diameters; dynamic light scattering ${ }^{a}: z$-average diameters). For monodisperse samples $\left(M_{\mathrm{w}} / M_{\mathrm{n}} \approx 1\right)$, average values will be similar for all of the calculations whereas for a typical polydisperse sample values can vary by several orders of magnitude (cf. Figure 4)

\begin{tabular}{llrl}
\hline & Number-average & Weight-average $^{b}$ & $z$-Average \\
\hline Molar mass & $M_{\mathrm{n}}=\frac{\sum n_{i} M_{i}}{\sum n_{i}}$ & $M_{\mathrm{w}}=\frac{\sum n_{i} M_{i}^{2}}{\sum n_{i} M_{i}}$ & $M_{z}=\frac{\sum n_{i} M_{i}^{3}}{\sum n_{i} M_{i}^{2}}$ \\
Diameter & $d_{\mathrm{n}}=\frac{\sum n d_{i}}{\sum n}$ & $d_{\mathrm{w}}=\frac{\sum n_{i} d_{i}^{4}}{\sum n_{i} d_{i}^{3}}$ & $d_{z}=\frac{\sum n_{i} d_{i}^{6}}{\sum n_{i} d_{i}^{5}}$ \\
& $d_{\mathrm{n}}=\frac{\sum w_{i} / d_{i}}{\sum w_{i} / d_{i}^{2}}$ & $d_{\mathrm{w}}=\frac{\sum w_{i} d_{i}}{\sum w_{i}}=\frac{\sum n_{i} d_{i} M_{i}}{\sum n_{i} M_{i}}$ & $d_{z}=\frac{\sum c_{i} d_{i} M_{i}}{\sum c_{i} M_{i}}$ \\
\hline
\end{tabular}

${ }^{a}$ Dynamic light scattering calculates the $z$-average diameter for Rayleigh scatterers $(d \ll \lambda)$ and for particles where measurements have been extrapolated to a scattering angle of 0 .

${ }^{b}$ Calculations of diameter averages assume that $n_{i} d^{3}$ is proportional to the weight of the particles through the density and that the density is constant for all size ranges.

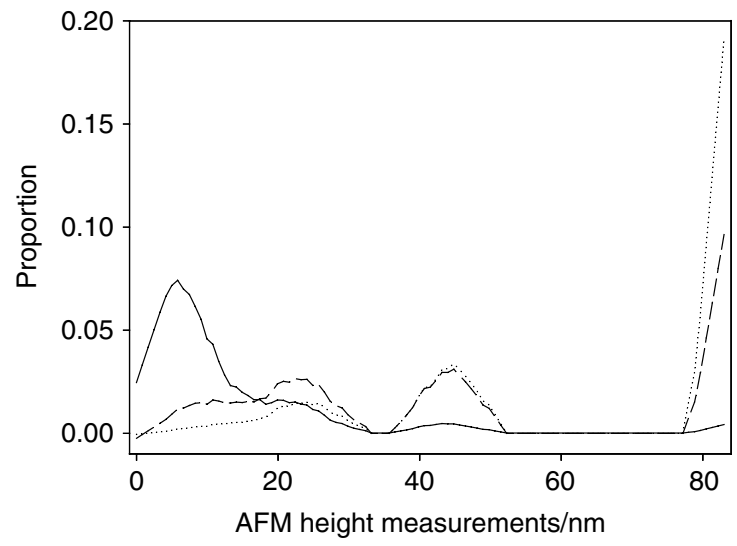

Figure 4. Number-(solid line), weight-(dashed line) and $z$-average (dotted line) diameters calculated from the AFM colloidal size (heights) distribution given in Figure 2a using the equations provided in Table 1. In this case of a natural, polydisperse sample, the number-average mean was calculated as $12.2 \mathrm{~nm}$, the weight-average diameter as $58.5 \mathrm{~nm}$ and the $z$-average diameter as $75.8 \mathrm{~nm}$. The example illustrates that even for the same sample, techniques that are based on different principles will provide a substantially different indication of the particle size distributions. In addition, the analytical bias of the technique (e.g. detection window, Figure 1) will significantly influence what is recorded by the scientist. To facilitate comparison, data have been smoothed using a negative exponential

processed or model systems under controlled laboratory conditions. In order to overcome some of the major difficulties that remain in this field, adequate and reliable methods for sampling, sample handling, fractionation, analysis and modelling will need to be developed. 
Finally, when considering the size of a colloid or particle of particular shape, it is essential to ask two questions. First, why are the size-based measurements being performed, i.e. what does this analysis reveal about environmental processes such as trace element uptake to biota? Second, are there ways of performing more revealing analyses? The first question is idealistic and forces us to consider the link between size and the relevant processes. There is no doubt that size is a useful parameter for considering colloidal behaviour. It affects processes such as the transport and biouptake of trace pollutants in sediments, soils, waters and the atmosphere. Furthermore, colloidal size is a standard parameter that can, with reservations, be used to compare data sets. Nevertheless, the reliance on size as a primary measurement is limiting, as discussed in the next section. The second question is more practical. More revealing parameters can indeed be measured. A significant current challenge in the study of environmental colloids and particles is to develop these methods and to apply them in order to produce non-trivial results that are based on parameters other than size.

\section{NON-SIZE-BASED MEASUREMENTS OF COLLOIDS AND PARTICLES}

Although size is a useful and frequently measured parameter, it is limited in the extent to which it can be used to gain detailed understanding of environmental behaviour. For instance, larger particles often dominate the sedimentation process. Since the larger particles generally, but not always [36,37], dominate particle mass distributions [1], a mass distribution may be the most relevant means to present sedimentation data. In contrast, insight into aggregation processes will likely benefit more from considering particle number distributions, which are likely to be dominated by the smaller colloids. Finally, surface area may be the most relevant parameter when considering pollutant uptake by colloids and particles. Like particle number, surface area is likely to be dominated by smaller colloids, albeit not in an identical manner. Since these three processes, i.e. uptake, aggregation and sedimentation, are interrelated, e.g. through the colloidal pumping model [38], a thorough understanding of the entire process will likely require accurate determinations of the mass, number and specific surface area distributions.

For a number of processes, size can be considered as a proxy for other parameters. In particular, knowledge of the diffusion coefficients of colloidal complexes with trace elements and organic pollutants is vital to understanding: (i) their transport in soils, sediments and the diffusive boundary layer around surfaces; (ii) their mass transport to biological organisms [39] and (iii) their quantitative analytical determination using important in situ metal speciation techniques such as diffusive gradients in thin films (DGT) and voltammetry. Although a number of techniques measure colloidal diffusion coefficients (Table 2), data are most often converted to size, as in the case of FlFFF [21,22,40] by assuming that the colloids are impermeable and spherical (Stokes-Einstein equation). Clearly, in natural systems, there is a range of colloidal morphologies, many of extreme complexity. Although the conversion to equivalent radii is helpful for making comparisons, diffusion coefficients are arguably a more powerful indicator of colloidal activity (aggregation, reactivity, etc.) and should be retained, whenever possible. In addition, other parameters such as fractal dimensions (aggregates) [41,42], colloidal form factors [24], gyration radii or contour or persistence lengths (e.g. fibrils) [43] may also provide structural information that is more easily related to environmental function. Similar arguments are valid 
Table 2. Comparison of most probable (mean or median) diffusion coefficients $\left(\mathrm{m}^{2} \mathrm{~s}^{-1} \times 10^{10}\right)$ for the Suwannee River standard fulvic acid. Figures in parentheses are standard deviations. For FCS, poor accuracy was obtained at high ionic strength $(n=3)$; for FlFFF, no values were measurable at low $\mathrm{pH}$ and high ionic strength $(n=3)$; for PFG NMR, data for regions 1-4 (corresponding to $0.8-1.9,1.9-3.5,3.5-4.3,6.3-8.1 \mathrm{ppm}$ ) have been collapsed to give the most probable diffusion coefficients. For PFG-NMR, standard deviations for all individual data in all cases were 0.2. Reprinted with permission from Environmental Science and Technology, 34, 3508-33513. Copyright 2000 American Chemical Society

\begin{tabular}{lcccc}
\hline & & \multicolumn{3}{c}{$\mathrm{pH}$ or $\mathrm{pD}$} \\
\cline { 3 - 5 } & Ionic strength $/ \mathrm{mmol} \mathrm{dm}^{-3}$ & 4 & 5.5 & 7.0 \\
\hline FCS & 5 & $2.21(0.07)$ & $2.52(0.02)$ & $2.71(0.06)$ \\
& 50 & $2.05(0.02)$ & $2.40(0.04)$ & $2.61(0.04)$ \\
FIFFF & 500 & $2-3$ & $2-3$ & $2-3$ \\
& 5 & - & $3.0(0.08)$ & $2.9(0.03)$ \\
PFG-NMR & 50 & - & $1.9(0.05)$ & $2.2(0.02)$ \\
& 500 & - & & \\
& Low & $2.8-3.6$ & $2.5-3.7$ & $2.4-3.5$ \\
& 500 & $2.6-3.8$ & $2.5-3.7$ & $2.5-3.5$ \\
\hline
\end{tabular}

for other colloidal characterisation techniques such as electrophoresis [34], which provides an estimate of the charge/size ratio of the colloids [34,44], although conversions of electrophoretic mobilities often require significant and complex interpretation [45].

\section{STRATEGIES FOR ADVANCING OUR UNDERSTANDING OF COLLOIDAL SYSTEMS}

Based on the above considerations, it is clear that to enhance further our understanding of environmental colloids and their impacts, there is a need to (i) employ in situ methods [46]; (ii) use non-perturbing methods [7]; (iii) use a variety of methods for comparative purposes $[23,27,28,47]$; and (iv) take great care in sampling and sample processing and use appropriate checks including the measurement of standard materials, where appropriate [48]. The use of standard materials is discussed in Chapter 3 in relation to ultrafiltration and cross-flow filtration (CFF) techniques, but is nevertheless a continuing limitation in the nanoparticle range where few appropriate standards are available [24,25]. The simultaneous use of a number of methods is another key point. Indeed, a number of studies have demonstrated that a reliance on any single technique may introduce substantial distortions of our view of the colloidal structure [15,28,33,36,47] and their effects on pollutant uptake [10]. When analysing the same colloidal sample, agreement between several techniques will provide increased confidence in the result [28] while disagreement will require the differences to be rationalised appropriately. This type of comparison can also mitigate the analytical uncertainties that normally occur when analysing unknown samples in the absence of certified reference materials.

An example that demonstrates the importance of using several techniques in parallel is given in Table 2. For diffusion coefficients of the reasonably monodisperse and purified Suwannee River standard fulvic acid, good agreement was obtained by FCS, nuclear magnetic resonance (NMR) and FlFFF [28]. In contrast, some multi-method studies to 
evaluate colloidal properties have shown only partial agreement (and indeed often substantial differences) in size distributions, metal binding properties and colloidal morphologies, especially for natural samples $\{$ e.g. AFM, transmission electron microscopy (TEM) and FCS [49]; AFM and ESEM [15]; AFM and TEM [47]; and CFF and FlFFF [10]\}. In addition to the size distributions provided in Figure 2, comparative SEM and ESEM images obtained from a single sampling from a freshwater sample are given in Figure 4. Substantially different conformations and surface coverages are shown. Similarly, for the same site, AFM images [15] were very different from EM images. Interestingly, TEM and AFM images were generally more similar [47] than images from AFM and ESEM, even though the experimental conditions were nearly identical for the AFM and ESEM acquisition (i.e. relative humidity of $c a$. 50-60\%). Although TEM images are generally obtained under high vacuum, the use of hydrophilic resins and multi-method TEM sample preparation techniques [24] may be sufficient to stabilise the three-dimensional structure of the colloids and colloidal aggregates [23]. In a final example that clearly demonstrates the complementary nature of three colloidal characterisation techniques, intrinsic viscosity measurements convincingly demonstrated an increase in the diameter of humic substances with increasing $\mathrm{pH}$ [50] whereas FCS demonstrated the opposite trend [28] and AFM height measurements showed no trend [26]. In this case, the differences occurred because each of the techniques probed different parameters. The story of the blind men trying to define an elephant by touch may be useful here. One man finds the tail and thinks it is a snake, another finds a leg and thinks it is a tree and so on. Similarly, in the above example, the intrinsic viscosity measurements evaluated molecular volumes (but ignored aggregates) whereas FCS measured an average diffusion coefficient that takes into account the effect of aggregation in solution. The AFM measurements evaluated adsorbed (molecular or aggregate) heights following interaction with a substrate (and possible reorganisation at the solid-water interface).

Clearly, the analytical uncertainty associated with multi-method measurements of natural systems is increased because it is difficult to know a priori whether the analyses are incorrect or rather whether different aspects of the same structure have been revealed. The development of certified reference materials, although difficult due to colloidal complexity and instability, may nonetheless be helpful for checking instrument operation. In addition, relevant materials, including synthetic manganese or iron oxides, standard humic substances (HS; e.g. International Humic Substances Society standards) or microbial exudates are being used more often and with greater success. Future research will therefore require the investigation of 'standard' or reference colloids in addition to minimally perturbed colloids from real systems.

\section{FUTURE PERSPECTIVES}

Over the past 15 years, enormous progress has been made towards an understanding of environmental colloidal systems, including the development and application of fractionation and analysis techniques; the development of models; the elucidation of colloidal structure and their interaction with trace elements, nutrients and pathogens; and the impact of colloids on the fate and behaviour of the trace elements, nutrients and pathogens.

The development of powerful techniques by which colloids and particles can be fractionated and analysed has been the primary, unambiguous improvement of recent years. 
As detailed in the chapters that follow, the (continuing) development and optimisation of novel, minimally perturbing (ideally in situ) methods has immensely improved our ability to quantify colloidal and particulate structures and their behaviour in the environment. In addition, sophisticated models for quantifying colloidal structure and predicting pollutant binding to colloids and colloidal transport have been developed. Nevertheless, further improvements are still required before the majority of models can be used with confidence in the real environment. Hence the iterative development and application of techniques and models to real systems is a key future development. Further discussion of this point is found in each of the individual chapters, although it should be noted that many of the most recent methods have only rarely been used on unperturbed colloidal environmental samples.

Significant developments into the analysis of colloidal structure and their impact on pollutant, nutrient and pathogen fate and behaviour have occurred in the last 15 years, although this field is also still at an early stage. Many of the colloidal characterisation techniques (AFM, ESEM, FCS, CE, FFF, etc.) have not been fully or appropriately deployed in the environment and therefore our current understanding of natural colloidal structures is tentative and only partially quantitative. Significant improvements are expected over the next 15 years once we are better able to couple the nanoscopic characterisation obtained by using FlFFF, AFM, TEM and other methods with conceptual models of colloid structure and their interactions. A more systematic determination of parameters, such as diffusion coefficients and fractal dimensions, will also be useful to gain greater understanding at a fundamental, mechanistic and quantitative level. Our understanding of colloidal systems has greatly evolved since the production of the first volume of Environmental Particles [1], 15 years ago. The next 15 years are certain to be extremely promising with exciting developments of fundamental knowledge in this vital area.

\section{REFERENCES}

1. Buffle, J. and van Leeuwen, H. P., eds (1992). Environmental Particles. IUPAC Series on Analytical and Physical Chemistry of Environmental Systems, Vol. 1. Lewis, Boca Raton, FL.

2. Goodwin, J. (2004). Colloids and Interfaces with Surfactants and Polymers. John Wiley \& Sons, Ltd, Chichester.

3. Dowling, A. (2005). Nanoscience and Nanotechnologies: Opportunities and Uncertainties. Royal Society/Royal Academy of Engineering, London.

4. Lead, J. R., Davison, W., Hamilton-Taylor, J. and Buffle, J. (1997). Characterizing colloidal material in natural waters Aquat. Geochem., 3, 213-232.

5. Guo, L. and Santschi, P. H. (2006). Ultrafiltration and its application to sampling and Characterisation of aquatic colloids. In Environmental Colloids and Particles: Behaviour, Structure and Characterisation, eds Wilkinson, K. J. and Lead, J. R. IUPAC Series on Analytical and Physical Chemistry of Environmental Systems, Vol. 10. John Wiley \& Sons, Ltd, Chichester, Chapter 4.

6. Gustafsson, O. and Gschwend, P. M. (1997). Aquatic colloids: concepts, definitions, and current challenges, Limnol. Oceanogr., 42, 519-528.

7. Stumm, W. (1993). Aquatic colloids as Chemical reactants: surface structure and reactivity, Colloids Surf. A, 73, 1-18.

8. Buffle, J. and Leppard, G. G. (1995). Characterization of aquatic colloids and macromolecules: Part I. Structure and behaviour of colloidal material, Environ. Sci. Technol., 29, 2169-2175. 
9. Doucet, F. J., Lead, J. R. and Santschi, P. H. (2006). Colloids-trace element interactions in aquatic systems. In Environmental Colloids: Behaviour, Structure and Characterisation, eds Wilkinson, K. J. and Lead, J. R. IUPAC Series on Analytical and Physical Chemistry of Environmental Systems, Vol. 10. John Wiley \& Sons, Ltd, Chichester, Chapter 3.

10. Benedetti, M. F., Ranville, J. F., Allard, T. and Bednar, A. J. (2003). The iron status in colloidal matter from the Rio Negro, Brazil, Colloids. Surf. A, 217, 1-9.

11. Foster, A. L., Brown, G. E. and Parks, G. A. (2003). X-ray absorption fine structure study of $\mathrm{As}(\mathrm{V})$ and $\mathrm{Se}(\mathrm{IV})$ sorption complexes on hydrous Mn oxides, Geochim. Cosmochim. Acta, 67, 1937-1953.

12. Campbell, P. G. C., Twiss, M. R. and Wilkinson, K. J. (1997). Accumulation of natural organic matter on the surfaces of living cells: implications for the interaction of toxic solutes with aquatic biota, Can. J. Fish. Aquat. Sci., 54, 2543-2554.

13. Gustafsson, O., Duker, A., Larsson, J., Andersson, P. and Ingri, J. (2000). Functional separation of colloids and gravitoids in surface waters based on differential settling velocity: coupled cross-flow filtration-split flow thin-cell fractionation (Cff-Splitt), Limnol. Oceanogr., 48, 1731-1742.

14. Heathwaite, L., Haygarth, P., Matthews, R., Preedy, N. and Butler, P. (2005). Evaluating colloidal phosphorus delivery to surface waters from diffuse agricultural sources, J. Environ. Qual., 34, 287-298.

15. Doucet, F. J., Maguire, L. and Lead, J. R. (2004). Size fractionation of aquatic colloids and particles by cross flow filtration: analysis by scanning electron and atomic force microscopy, Anal. Chim. Acta, 522, 59-71.

16. Horowitz, A. J., Lum, K. R., Garbarino, J. R., Hall, G. E., Lemieux, C. and Demas, C. R. (1996). Problems associated with using filtration to define dissolved trace element concentrations in natural water samples, Environ. Sci. Technol. 30, 954-963.

17. Buffle, J., Perret, D. and Newman, M. (1992). The use of filtration and ultrafiltration for size fractionation of aquatic particles. In Environmental Particles, Vol. 1, eds Buffle, J. and van Leeuwen, H. P. Lewis, Boca Raton, FL, p. 554.

18. Sutton, R. and Sposito, G. (2005). Molecular structure in soil humic substances: the new view, Environ. Sci. Technol., 39, 9009-9015.

19. Gimbert, L. J., Haygarth, P. M., Beckett, R. and Worsfold, P. J. (2005). Comparison of centrifugation and filtration techniques for the size fractionation of colloidal material in soil suspensions using sedimentation field-flow fractionation, Environ. Sci. Technol., 39, 1731-1735.

20. Doucet, F. J., Lead, J. R., Maguire, L., Achterberg, E. and Millward, G. (2005). Visualisation of natural aquatic colloids and particles - a comparison of conventional high vacuum SEM and environmental scanning electron microscopy, J. Environ. Monit., 7, 115-121.

21. Hassellöv, M., von der Kammer, F. and Beckett, R. (2006). Characterisation of aquatic colloids in macromolecules by field-flow fractionation. In Environmental Colloids: Behaviour, Structure and Characterization, eds Wilkinson, K. J. and Lead, J. R. IUPAC Series on Analytical and Physical Chemistry of Environmental Systems, Vol. 10. John Wiley \& Sons, Ltd, Chichester, Chapter 5.

22. Lyven, B., Hassellov, M., Turner, D. R., Haraldsson, C. and Andersson, K. (2003). Competition between iron- and carbon-based carriers for trace metals in a freshwater assessed using flow field-flow fractionation coupled to ICP-MS, Geochim. Cosmochim. Acta, 67, 3791-3802.

23. Liss, S. N., Droppo, I. G., Flanagan, S. T. and Leppard, G. G. (1996). Floc architecture in wastewater and natural riverine systems, Environ. Sci. Technol., 30, 680-686.

24. Mavrocordatos, D., Perret, D. and Leppard, G. G. (2007). Strategies and advances in the characterisation of environmental colloids by electron microscopy. In Environmental Colloids: Behaviour, Structure and Characterisation, eds Wilkinson, K. J. and Lead, J. R., IUPAC Series on Analytical and Physical Chemistry of Environmental Systems, Vol. 10. John Wiley \& Sons Ltd, Chichester, Chapter 8.

25. Balnois, E., Papastavrou, G. and Wilkinson, K. J. (2006). Force microscopy and force measurements of environmental colloids. In Environmental Colloids: Behaviour, Structure and Characterisation, eds Wilkinson, K. J. and Lead, J. R. IUPAC Series on Analytical and Physical Chemistry of Environmental Systems, Vol. 10. John Wiley \& Sons, Ltd, Chichester, Chapter 9. 
26. Balnois, E., Wilkinson, K. J., Lead, J. R. and Buffle, J. (1999). Atomic force microscopy of humic substances: effects of $\mathrm{pH}$ and ionic strength, Environ. Sci. Technol., 33, 1311-1317.

27. Lead, J. R., Wilkinson, K. J. and Starchev, K. (2003). Diffusion coefficients of humic substances in agarose gel and in water, Environ. Sci. Technol., 37, 482-487.

28. Lead., J. R., Wilkinson, K. J., Balnois, E., Cutak, B., Larive, C., Assemi, S. and Beckett, R. (2000). Diffusion coefficients and polydispersities of the Suwannee River fulvic acid: comparison of fluorescence correlation spectroscopy, pulsed-field gradient nuclear magnetic resonance and flow field-flow fractionation, Environ. Sci. Technol., 34, 3508-3513.

29. Fatin-Rouge, N. and Buffle, J. (2007). Study of environmental systems by means of fluorescence correlation spectroscopy. In Environmental Colloids and Particles: Behaviour, Structure and Characterisation, eds Wilkinson, K. J. and Lead, J. R. IUPAC Series on Analytical and Physical Chemistry of Environmental Systems, Vol. 10. John Wiley \& Sons, Ltd, Chichester, Chapter 11.

30. Schurtenberger, P. and Newman, M. E. (1993). Characterization of biological and environmental particles using static and dynamic light scattering. In Environmental Particles, eds Buffle, J. and van Leeuwen, H. P. IUPAC Series on Analytical and Physical Chemistry of Environmental Systems, Vol. 2. Lewis, Boea Raton, FL.

31. Schmitt-Kopplin, P. and Junkers, J. (2006). Modern electrophoric techniques for the characterisation of natural Organic matter. In Environmental Colloids: Behaviour, Structure and Characterisation, eds Wilkinson, K. J. and Lead, J. R. IUPAC Series on Analytical and Physical Chemistry of Environmental Systems, Vol. 10. John Wiley \& Sons, Ltd, Chichester, Chapter 6.

32. Buffle, J. (1998). Complexation Reactions in Aquatic Systems: an Analytical Approach. Ellis Horwood, New York.

33. Droppo, I. G., Flannigan, D. T., Leppard, G. G., Jaskot, C. and Liss, S. N. (1996). Floc stabilization for multiple microscopic techniques, Appl. Environ. Microbiol. 62, 3508-3515.

34. Duval, J., Wilkinson, K. J., Buffle, J. and van Leeuwen, H. P. (2005). Humic substances are soft and permeable: evidence from their electrophoretic mobilities, Environ. Sci. Technol. 39, 6435-6445.

35. Villalobos, M., Bargar, J. and Sposito, G. (2005). Mechanisms of $\mathrm{Pb}(\mathrm{II})$ sorption on a biogenic manganese Oxide, Environ. Sci. Technol., 39, 569-576.

36. Lead, J. R., Davison, W., Hamilton-Taylor, J. and Harper, M. (1999). Trace metal sorption by natural particles and coarse colloids, Geochim. Cosmochim. Acta, 63, 1661-1670.

37. Moran, S. B. and Moore, R. M. (1989). The distribution of colloidal aluminium and organic carbon in coastal and open ocean waters off Nova Scotia, Geochim. Cosmochim. Acta, 53, 2519-2527.

38. Honeyman, B. D. and Santschi, P. H. (1992). The role of particles and colloids in the transport of radionuclides and trace metals in the oceans. In Environmental Particles, Vol. 1, (Buffle, J. and van Leeuwen, H. P., eds.), pp. 554, Lewis Publishers.

39. Wilkinson, K. J. and Buffle, J. (2004). Critical evaluation of physicochemical parameters and processes for modeling the biological uptake of trace metals in environmental (aquatic) systems. In Physicochemical Kinetics and Transport at Chemical-Biological Interphases, eds van Leeuwen, H. P. and Koester, W. John Wiley \& Sons, Ltd, Chichester, pp. 533.

40. Thang, N. M., Geckeis, H., Kim, J. I. and Beck, H. P. (2001). Application of the flow fieldflow fractionation (fFFF) to the characterization of aquatic humic colloids: evaluation and optimization of the method. Colloids Surf. A, 181, 289-301.

41. Redwood, P. S., Lead, J. R., Harrison, R. M. and Stoll, S. (2005). Characterization of humic substances by environmental scanning electron microscopy, Environ. Sci. Technol., in press.

42. Rizzi, F. R., Stoll, S., Senesi, N. and Buffle, J. (2004). A transmission electron microscopy study of the fractal properties and aggregation processes of humic acids, Soil Sci., 169, 765-775.

43. Balnois, E. and Wilkinson, K. J. (2002). Sample preparation techniques for the observation of environmental biopolymers by atomic force microscopy, Colloids Surf. A, 207, 229-242.

44. Radko, S. P. and Charambach, A. (2002). Separation and characterization of sub-mm- and mm-sized particles by capillary zone electrophoresis, Electrophoresis, 23, 1957-1972. 
45. Duval, J. F. L. (2006). Electrophoresis of soft colloids: basic principles and applications. In Environmental Colloids and Particles: Behaviour, Structure and Characterisation, eds Wilkinson, K. J. and Lead, J. R. IUPAC Series on Analytical and Physical Chemistry of Environmental Systems, Vol. 10. John Wiley \& Sons, Ltd, Chichester, chapter 7.

46. Buffle, J. and Horvai, G. (2000). In-Situ Monitoring of Aquatic Systems. John Wiley \& Sons, Ltd, Chichester.

47. Wilkinson, K. J., Balnois, E., Leppard, G. G. and Buffle, J. (1999). Characteristic features of the major components of freshwater colloidal organic matter revealed by transmission electron and atomic force microscopy, Can. J. Fish. Aquat. Sci., 155, 287-310.

48. Muirhead, D. and Lead, J. R. (2003). Physico-chemical characteristics of natural colloids in a heavily-polluted, urban watershed: analysis by atomic force microscopy, Hydrobiology 454, $65-69$.

49. Lead, J. R., Balnois, E., Hosse, M., Menghetti, R. and Wilkinson, K. J. (1999). Characterization of Norwegian natural organic matter: size, diffusion coefficients and electrophoretic mobilities, Environ. Int., 25, 245-258.

50. Avena, M. J., Vermeer, A. W. P. and Koopal, L. K. (1999). Volume and structure of humic acids studied by viscometry $\mathrm{pH}$ and electrolyte concentration effects, Colloids Surf. A, 151, $213-224$. 
